Tropical Journal of Pharmaceutical Research March 2016; 15 (3): 651-655

ISSN: $1596-5996$ (print); 1596-9827 (electronic)

(C) Pharmacotherapy Group, Faculty of Pharmacy, University of Benin, Benin City, 300001 Nigeria.

All rights reserved.

Available online at http://www.tjpr.org

Original Research Article

http://dx.doi.org/10.4314/tjpr.v15i3.30

\title{
Application and Curative Effect of Micro-implant Anchorage in Orthodontics
}

\author{
Ronghe Zhang ${ }^{1 *}$, Xueyan Bai ${ }^{1}$, Weiwei Zhang ${ }^{1}$, Xiaotong Song ${ }^{1}$, Chunyan \\ Wang $^{2}$, Xinxin Gao ${ }^{1}$, Xubiao Tian ${ }^{1}$ and Fengzhen Liu ${ }^{1}$ \\ ${ }^{1}$ Orthodontics Department, ${ }^{2}$ Department of Stomatology, Binzhou Medical University Hospital, Binzhou, Shandong, 256603, \\ China
}

*For correspondence: Email: zhangronghe01@sina.com

Revised accepted: 4 February 2016

\begin{abstract}
Purpose: To explore and analyze the curative effects of micro - implant anchorages in orthodontics. Methods: A retrospective analysis of 65 patients undergoing orthodontic treatment in Department of Stomatology, Binzhou Medical University Hospital, Shandong, China was carried out. Thirty four cases in the treatment group were treated with a micro-implant as the anchorage, while 31 cases of the control group were treated with a palatal bar and facebow as the anchorage, and the curative results of the two groups were then compared.

Results: After a 13-month treatment, both anchorages were clinically effective, but the micro-implant anchorage showed higher efficacy. Measurement indices for the test group, including sella-nasion - $A$ point (SNA) angle (- $1.88 \pm 0.71)$, sella-nasion-B point (SNB) angle (1.39 \pm 0.42$), A$ point - nasion - $B$ point (ANB) angle (- 2.40 \pm 0.83$)$, upper central incisor - lower central incisor (U1 - L1) angle (25.79 \pm 5.90), upper central incisor - sella - nasion (U1 - SN) angle (- $10.13 \pm 3.68)$, lower central incisor mandibular plane (L1 - MP) angle (- 4.22 \pm 0.45 ), upper central incisor - nasion - $A$ point (U1 - NA) angle $(-1.32 \pm 1.35)$ and lower central incisor - nasion - B point ( $L 1-N B)$ angle $(-1.32 \pm 1.35)$ of the test group were significantly different those of the control group Overbite (OB), overjet (OJ), intercanine width and the width of the first molars of treatment and control groups were also remarkably). Moreover, micro-implant was observed to be more stable during treatment.

Conclusion: Compared with traditional anchorages, micro-implants possess the advantages of slighter trauma, simpler operation, more reliable curative effect and high stability.
\end{abstract}

Keywords: Micro-implant anchorage, Orthodontics, Facebow anchorage, Oral medicine, Clinical efficacy

Tropical Journal of Pharmaceutical Research is indexed by Science Citation Index (SciSearch), Scopus, International Pharmaceutical Abstract, Chemical Abstracts, Embase, Index Copernicus, EBSCO, African Index Medicus, JournalSeek, Journal Citation Reports/Science Edition, Directory of Open Access Journals (DOAJ), African Journal Online, Bioline International, Open-J-Gate and Pharmacy Abstracts

\section{INTRODUCTION}

With the continuous development of society and great advancements in oral medicine, people require a better appearance of teeth and a few people have tried orthodontics. Orthodontics treats malformed teeth by exerting a continuous pressure on the teeth and facial skeleton, applying various types of regulation apparatus [1]. During treatment, every force exerted on the tooth generates an equal force but in the opposite direction, and the support used for this counteracting force is called an "anchorage" [2]. In fact, an anchorage is a foundation that provides the force to correct the teeth. Normally, an anchorage is composed of normal teeth, palate and phatnoma during orthodontics.

In recent years, micro - implant anchorage systems, a breakthrough technology, have been 
developed, benefiting from the advancements in materials science, on the basis of the achievements of prosthetics [3-5]. The clinical application and efficacy observation of micro implant anchorages have become a new research hot spot in the Department of Stomatology. Instead of taking teeth as the basis, as was previously the case, this new anchorage exerts the counteracting force of orthodontics on the jaw, thus completely avoiding the displacement of teeth. It solves a lot of problems existing in clinical anchorage. Micro - implant anchorages feature the efficacy that conventional methods cannot achieve. Moreover, it has attracted extensive attention for its advantages of slighter trauma, simpler operation, steadiness, reliable efficacy and limited dependence on the patient [6]. To explore and analyze the application and curative effects of micro - implant anchorages in orthodontics, this study carried out a retrospective analysis on clinical materials regarding 65 patients undergoing orthodontic treatment in our department, in 2012, among which 34 treatment group patients received treatment with micro - implant anchorages, while 31 control group patients were treated with palatal bar and facebow anchorages. The results of two groups were reported below.

\section{EXPERIMENTAL}

Sixty-five patients with maxillary protrusion undergoing orthodontic treatment were selected. A total of 34 treatment group patients were treated with micro - implants as anchorages, while 31 control group patients were treated with palatal bar and facebow anchorages.

\section{Clinical features}

None of the patients had an orthodontic history or systemic diseases and their oral hygiene was fine. The teeth of the patients were convex in side view. The correction scheme provided for the extraction of four first premolars, or the first premolar of the maxilla or the second premolar of the lower jaw. The patients were randomly divided into treatment group and control group, according to their admission time. There were 34 patients in the treatment group, 18 males and 16 females, aged from 12 to 26 years(mean 21.3 years), and 31 patients in the control group, 15 males and 16 females, aged from 14 to 25 years (mean 20.6 years). No statistical significance was found in age, gender, disease type and clinical performance etc. between the two groups. This study was approved by the medical ethics committee of Binzhou Medical University Hospital, Shandong, China and all the patients enrolled in gave their informed consent.

\section{Therapeutic methods}

\section{Treatment group}

Implantation of the micro implant: the teeth which required implanting were separated with copper wire, and the implant position was marked; then panoramic and periapical radiographs were made; the form and position of the fangs and the adjacent tissue were examined; the periosteal flap was not carried out, and a longitudinal incision of 3 - $5 \mathrm{~mm}$ was required, if the implanting position was covered by floppy phatnoma mucosa; the implanting position was normally $2-3 \mathrm{~mm}$ at the binding site of the membrane and gingiva, or $2-3 \mathrm{~mm}$ towards the root of the tooth, and the implanting level was almost perpendicular to the facial bones. The root tip was shut after the operation to confirm the link between micro - implant and root of the tooth. After the operation, the patients were prescribedan oral antibiotic in case of infection and, meanwhile, required to keep the oral cavity clean.

\section{Application of force on the micro-titanium screw}

A chain rubber ring, or tension spring, was used to exert a force between the implant and draw hook, after the implantation of the micro implant, which is normally applied; patients should then return on a monthly basis to change the chain rubber ring and tension spring.

\section{Removal of the microtitanium screw}

The top of the titanium screw was entangled with a manual screw tap and rotated counterclockwise, and the screw was then removed. Local infiltration anesthesia was unnecessary and the wound would heal in a couple of days without special processing.

\section{Control group}

In the case of the control group, a facebow was used as anchorage combined with an intraoral transpalatal bar. The facebow was worn for 8 to 12 hours a day and the traction was 200 to $300 \mathrm{~g}$ on each side. The other operations were the same as the treatment group.

\section{Observational index}

The main observational indexes were: sella nasion - A point (SNA) angle; sella - nasion - B point (SNB) angle; A point - nasion - B point (ANB) angle; upper central incisor - sella - nasion (U1 - SN) angle; lower central incisor-mandibular 
plane (L1 - MP) angle; upper central incisor lower central incisor (U1 - L1) angle; upper central incisor - nasion - A point (U1 - NA) angle; lower central incisor - nasion - B point (L1 - NB) angle; $\mathrm{OB}$ (overbite); OJ (overjet); intercanine width; width of the first molars.

\section{Statistical analysis}

A statistical analysis was performed using SPSS 19.0 software, the measurement data was expressed as mean \pm standard deviation (mean $\pm \mathrm{SD}$ ), and measurement materials was calculated with a pared-sample T-test. All data was considered statistically significant if $p<0.05$.

\section{RESULTS}

After a 13-month treatment period, both anchorages were clinically effective, while the micro - implant anchorage revealed a better performance. The micro - implants used in treatment group were of good stability overall, but some patients developed mild edema in soft tissues around micro-plant.

\section{Result comparison of cephalometrics}

The variation of the cephalometrics index of the treatment and control groups is shown in Table 1.

The cephalometric differences, SNA, SNB, ANB, U1 - L1, U1 - SN, L1 - MP, U1 - NA, L1 - NB angle of the micro - implant group and facebow group, before and after treatment, were of statistical significance $(p<0.05)$.

\section{Comparison of the model measurement results}

The comparison of the variations of the model measurement indexes, between the treatment and control groups, is shown in Table 2 .

\section{Steadiness of the micro-implant}

The molar displacement of the treatment group was $(3.60 \pm 0.41) \mathrm{mm}$, while the molar displacement of the control group was $(5.18 \pm$ $0.72) \mathrm{mm}$, and the difference was of statistical significance, which suggests that the microimplant was steady during the orthodontic treatment.

Table 1: Analysis of the cephalometrics of the micro-implant and facebow before and after treatment (mean \pm SD)

\begin{tabular}{|c|c|c|c|c|}
\hline \multirow{2}{*}{ Group } & \multicolumn{2}{|c|}{ Change } & \multirow{2}{*}{ T-value } & \multirow{2}{*}{$P$-value } \\
\hline & $\begin{array}{c}\text { (Treatment } \\
\text { group) }\end{array}$ & (Control group) & & \\
\hline $\begin{array}{l}\text { SNA angle } \\
\text { (degree) }\end{array}$ & $-1.88 \pm 0.71$ & $-0.73 \pm 0.64$ & 6.776 & $<0.05$ \\
\hline $\begin{array}{l}\text { SNB angle } \\
\text { (degree) }\end{array}$ & $1.39 \pm 0.42$ & $0.71 \pm 0.29$ & 9.008 & $<0.05$ \\
\hline $\begin{array}{l}\text { ANB angle } \\
\text { (degree) }\end{array}$ & $-2.40 \pm 0.83$ & $-1.33 \pm 0.68$ & 5.653 & $<0.05$ \\
\hline $\begin{array}{l}\text { U1-L1 angle } \\
\text { (degree) }\end{array}$ & $25.79 \pm 5.90$ & $10.30 \pm 3.05$ & 13.102 & $<0.05$ \\
\hline $\begin{array}{l}\text { U1-SN angle } \\
\text { (degree) }\end{array}$ & $-18.80 \pm 1.94$ & $-10.13 \pm 3.68$ & 12.073 & $<0.05$ \\
\hline $\begin{array}{l}\text { L1-MP angle } \\
\text { (degree) }\end{array}$ & $-7.31 \pm 2.11$ & $-4.22 \pm 0.45$ & 7.985 & $<0.05$ \\
\hline $\begin{array}{l}\text { U1-NA angle } \\
(\mathrm{mm})\end{array}$ & $-5.80 \pm 1.74$ & $-2.83 \pm 1.12$ & 8.094 & $<0.05$ \\
\hline $\begin{array}{l}\text { L1-NB angle } \\
\text { (degree) }\end{array}$ & $-3.11 \pm 2.09$ & $-1.32 \pm 1.35$ & 8.802 & $<0.05$ \\
\hline
\end{tabular}

Note: SNA: sella - nasion - A point; SNB: sella - nasion - B point; ANB: A point - nasion - B point, U1 - SN: upper central incisor - sella - nasion; L1 - MP: lower central incisor - mandibular plane; U1 - L1: upper central incisor - lower central incisor; U1 - NA upper central incisor - nasion - A point; L1 - NB: lower central incisor nasion - B point 
Table 2: Model measurement variation of the micro - implant and facebow, before and after treatment (mean \pm SD)

\begin{tabular}{|c|c|c|c|c|}
\hline Group & Treatment group & $\begin{array}{l}\text { Control } \\
\text { qroup }\end{array}$ & T-value & $P$-value \\
\hline $\mathrm{OB}$ (degree) & $-4.49 \pm 1.03$ & $-3.84 \pm 1.32$ & 2.224 & $<0.05$ \\
\hline OJ(degree) & $-6.57 \pm 1.45$ & $-5.69 \pm 1.07$ & 8.733 & $<0.05$ \\
\hline Intercanine width (mm) & $1.71 \pm 0.83$ & $0.64 \pm 0.58$ & 5.969 & $<0.05$ \\
\hline $\begin{array}{l}\text { Width of the first } \\
\text { molars (mm) }\end{array}$ & $-1.48 \pm 0.54$ & $0.25 \pm 0.07$ & 12.578 & $<0.05$ \\
\hline
\end{tabular}

Measurement variations of the indexes of $O B, O J$, intercanine width and width between the first molars of the micro-implant and facebow group was found to be of statistical significance $(p<0.05)$

\section{DISCUSSION}

Malocclusion and malformation, along with caries and periodontal disease, are three common diseases of the oral cavity and its morbidity exceeds $50 \%$. Normally, in orthodontics, devices such as the intraoral group teeth, transpalatal arch, Nance bow, hyoid arch and some extraoral devices are applied [7]. In recent years, the micro-implant anchorage has become a new research hot spot in the field of stomatology, regarding the application and efficacy observation in orthodontics, for its advantages of steadiness, reliability, simple operation, slighter trauma, etc. The most remarkable difference between the micro - implant anchorage system and conventional anchorages is that its fixation is independent from synosteosis, but relies instead on the mechanical interlocking of the implant and the bone tissue [8-10].

The evaluation of the efficacy of orthodontic treatments applying conventional facebow anchorage depends on patient cooperation. But micro - implant anchorages can retract the anterior teeth by taking maximum advantage of tooth extraction diastema and improve the relation between facial form and molar, to achieve a perfect anchorage controlling effect $[11,12]$. Though both kinds of anchorages were clinically effective, a better performance of the micro - implants was observed. The comparison of the difference between the two groups, before and after the treatment, showed that the differences were statistically significant in U1 NA, U1 - SN, U1 - L1 and L1 - MP. It suggested that the molars were steadier and the incisor retrusion and intrusion was better in the treatment group.

A reduction of SNA of the treatment group was observed $(p<0.05)$, which appeared to be caused by a point retrusion resulting from a large-scale adduction of the dental crown and root tip of the upper central incisor. As the treatment of the control group required the cooperation of the patients, the adduction was restrained and there was no obvious variation of SNA. Meanwhile, SNB of the treatment group induced considerably $(p<0.05)$, and the upper teeth and lower teeth including OJ, OB, relation between canine teeth and molars and the incisor location, were distinctly improved; the width between the molars of the upper and lower jaw was obviously reduced in the treatment group, probably as a result of the automatic adaptive adjustment of the dental arch after tooth extraction; while due to the long-term wearing of the facebow there was no distinct improvement in the control group.

During orthodontic treatment, the cacodontia is expected to move in the right direction and for the appropriate distance, while the anchorage tooth is not supposed to move, to maintain healthy cooperative relation [13]. In this research, the molar displacement of the treatment group was $(3.60 \pm 0.41) \mathrm{mm}$, which was obviously reduced compared with the control group, and the difference was of statistical significance.

Though the micro-implant is small in size, easy to operate and steadier than the conventional implant, some points should be highlighted [14]. Firstly, treatment objectives are dominated by children who fear an operation, therefore the operation is highly required; secondly, orthodontics may cause some complications such as local infection, tooth root damage and to the adjacent tissues, such as teeth roots, nerves and blood vessels, during the movement of the micro-implant, therefore the implantation should be as far as possible from any nerves, blood vessels, tissue and organs; thirdly, the implanting position should be determined considering the 
width between the root tips of adjacent teeth, and the safe distance must at least $4.95 \mathrm{~mm}$.

\section{CONCLUSION}

Micro-implant acting as an anchorage in orthodontics can effectively improve the success rate of orthodontic treatment. Micro-implant treatment, which has the advantage of simple operation, minimal damage and good compliance, is superior to traditional orthodontic treatment; hence, it is worth adopting in clinical practice.

\section{REFERENCES}

1. Yin $X Y$. Application Value of Micro Implant Anchorage in Orthodontics. Chin Pract Med 2014; 9(12): 58-59.

2. Jin $A P, H u X C$, Huang $Y Y$. Micro-implant Anchorage in Orthodontics for Adults. Chin J Aesth Med 2012; 21(6): 1003-1004.

3. Tong ZY, Li TY. Analysis of the Application of Micro Implant Anchorage in Adult Orthodontics. Medi Aesth Bea 2013; 22(9): 61-62.

4. Wang XH, He XC. Application Effect of Micro Implant Anchorage in Orthodontics. Chin Med Eng 2014; 22(1): 86-87.

5. Yang FS, Yang S. Application Effect of Micro Implant Anchorage in Orthodontics. Chin J Mod Drug App 2014; 8(19): 64-65.
6. Wu XP, Li B, Feng YX. Combination Application Implant as Anchorage In of Implant and Mini-screw Orthodontic and Renovation. Chin J Oral Implan 2013; 18(4): 191193.

7. Chen ZC. Value of Micro-implant Anchorage Orthodontic Treatment. J Med Forum 2012; 32(24): 48-50.

8. Wei YM. Efficacy Analysis of Micro Implant Anchorage in Orthodontics. Med Forums in Basic 2012; 16(34): 45424543.

9. Tang HF. Clinical Application and Effects Observation of Micro-Implant Anchorage for 74 Cases of Orthodontics. Guide Chin Med 2010; 8(17): 41-42.

10. An K, Wan HZ, Xiao CF. Clinical Efficacy of Micro-implant Anchorage in Orthodontics Treatment. J Clin Med Pract 2013; 17(13): 71-72.

11. Huang $M Y$, Zhang J. The Clinical Effect of Implant Anchorage in Orthodontics Improving Gummy Smile. Jilin Med J 2013; 34(14): 2669-2670.

12. Feng $Y$. Application of Micro Implant Anchorage in Teenager Orthodontics. Hebei Med J 2013; 35(3): 352353.

13. Wu GY, Yang SJ. Application Value on Micro Implant Anchorage in the Treatment of Orthodontic. Med Innov Chin 2014; 11(7): 80-82.

14. Yu ZX. Application Discussion of Micro Implant Anchorage in Clinical Orthodontics. Chin Prescri Drug 2014; 12(7): 86-86. 